\title{
Recognizing obsessive-compulsive disorder: how suitable is the German Zohar-Fineberg obsessive-compulsive screen?
}

\author{
Franziska Kühne*, Tatjana Paunov and Florian Weck
}

\begin{abstract}
Background: Despite the prevalence of obsessive-compulsive disorder (OCD), its precise identification remains challenging. With the Zohar-Fineberg Obsessive-Compulsive Screen (ZF-OCS; 5 or 6 items), a brief instrument is widely available mainly in English. As there is a lack of empirical studies on the ZF-OCS, the aim of the present study was to translate the items into German and investigate the instrument in a nonclinical sample.

Methods: In two consecutive online surveys, $n=304$ and $n=51$ students participated. Besides the ZF-OCS, they answered established measures on OCD, depression, health anxiety, general anxiety and health-related well-being.

Results: Whereas internal consistency was low $(a=.53-.72 ; \omega=.55-.69)$, retest reliability $\left(r_{\mathrm{t} 1, \mathrm{t2}}=.89\right)$ at two weeks was high. As expected, we found high correlations with other OCD instruments ( $r>61$; convergent validity), and significantly weaker correlations with measures of depression $(r=.39)$, health anxiety $(r=.29)$, and health-related well-being ( $r=-.28$, divergent validity). Nonetheless, the correlations with general anxiety were somewhere in between $(r=.52)$.

Conclusions: Due to heterogeneous OCD subtypes, the ZF-OCS asks diverse questions which probably resulted in the present internal consistency. Nevertheless, the results on retest reliability and validity were promising. As for other OCD instruments, divergent validity regarding general anxiety seems problematic to establish. Even so, the ZF-OCS seems valuable for screening purposes, as it is short and easy to administer, and may facilitate initiating subsequent clinical assessment. Further studies should determine the instrument's diagnostic accuracy.
\end{abstract}

Keywords: Assessment, Obsessive-compulsive disorder, Psychodiagnostics, Psychometric properties, Questionnaire, Screening

\section{Background}

Obsessive-compulsive disorder (OCD) is characterized either by the presence of recurrent and persistent thoughts, urges or images perceived as intrusive and unwanted (obsessions) and/or by repetitive behaviors or mental acts that the individual feels driven to perform in response (compulsions) [1]. Obsessions and compulsions may refer to the symptom dimensions of a)

\footnotetext{
* Correspondence: dr.franziska.kuehne@uni-potsdam.de Department of Psychology, Clinical Psychology and Psychotherapy, University of Potsdam, Karl-Liebknecht-Str. 24-25, 14476 Potsdam, Germany
}

contamination, b) responsibility for harm, injury, or bad luck, c) symmetry, completeness, or exactness or d) violent, sexual or religious thoughts [2]. OCD is a prevalent disorder with a 12-month prevalence ranging between $1.2 \%$ in the US American [3] and 3.6\% in the German general population [4]. OCD substantially and negatively affects quality of life [5], social life, work and family life [6].

Despite the impairment associated with OCD, many years may pass until individuals receive adequate treatment [7]. For example, Wahl and colleagues [8]

(c) The Author(s). 2021, corrected publication 2022. Open Access This article is licensed under a Creative Commons Attribution 4.0 International License, which permits use, sharing, adaptation, distribution and reproduction in any medium or format, as long as you give appropriate credit to the original author(s) and the source, provide a link to the Creative Commons licence, and indicate if changes were made. The images or other third party material in this article are included in the article's Creative Commons licence, unless indicated otherwise in a credit line to the material. If material is not included in the article's Creative Commons licence and your intended use is not permitted by statutory regulation or exceeds the permitted use, you will need to obtain permission directly from the copyright holder. To view a copy of this licence, visit http://creativecommons.org/ licenses/by/4.0/. The Creative Commons Public Domain Dedication waiver (http://creativecommons.org/publicdomain/zero/1. 0/) applies to the data made available in this article, unless otherwise stated in a credit line to the data. 
determined that only $28 \%$ of outpatients diagnosed with OCD by the study team had received the correct diagnosis during a previous visit to their psychiatric practice. Not only in specialized settings, but also in primary care, under-recognition represents a considerable challenge. According to recent meta-analyses, the mean sensitivity of general practitioners to correctly diagnose anxiety and other mental disorders accounts for around $42-50 \%$ only [9]. This is highly relevant for OCD, as patients may present their symptoms to various physicians (e.g., due to fear of cancer or an infectious disease) or may overemphasize physical symptoms such as dermatitis resulting from excessive washing [10]. OCD patients may also withhold symptoms (such as religious or aggressive obsessions) because of shame, a prominent emotion in OCD [11]. Furthermore, they may describe other complaints such as depressive symptoms, traumatic life events, or family or sleeping problems to their physician [12] which then misleads the doctor.

Since under-recognition presents a problem for timely allocation to adequate treatment, several authors recommend routinely using screening questions during any mental state examination in specialized and in general health care settings $[8,13]$. Nevertheless, there is a lack of validated, brief OCD screening tools [14], especially in Germany [7]. One instrument that was proposed for active screening purposes is the Zohar-Fineberg Obsessive Compulsive Screen (ZF-OCS; [13]). It comprises the following five questions that take only a few minutes to go through: 1) "Do you wash or clean a lot?", 2) "Do you check things a lot?", 3) "Is there any thought that keeps bothering you that you'd like to get rid of but can't?", 4) "Do your daily activities take a long time to finish?", and 5) "Are you concerned ... either ... about orderliness and symmetry $[10,13]$ or ... about putting things in a special order or are you very upset by mess?" [14].

The ZF-OCS was validated in dermatology outpatients against the OCD item of the Mini-International Neuropsychiatric Interview [15], and it was found to have excellent sensitivity (94\%) and good specificity (85\%; [16]). Therefore, it was included in national guidelines and considered a promising screening tool $[7,14]$. Although the German Association for Psychiatry, Psychotherapy and Psychosomatics [7] offers a translation, no validation study has been published so far. To the best of our knowledge, the only available results were briefly published from a pilot study, and indicate excellent sensitivity and specificity, as well as good internal consistency of the tool [8]. In addition to the five ZF-OCS items, the authors of the pilot study used additional questions for the assessment of symptom severity and impairment, which however, they did not publish [8]. Accordingly, using additional questions was proposed by the national guideline as well [7], without specification of which questions to use.
The purpose of the current study was to investigate the reliability and validity of the German ZF-OCS in a non-clinical sample. We hypothesized that the ZF-OCS would show acceptable internal consistency as well as good retest reliability. We further expected evidence of convergent validity, i.e., high correlations with other OCD measures, and evidence of discriminant validity defined by weaker correlations with measures of anxiety, depression, and health-related well-being. Furthermore, we explored the psychometric properties of the ZF-OCS with and without an additional question on the individual impairment caused by current OC symptoms.

\section{Methods}

\section{Participants and procedure}

Participants were recruited at the University of Potsdam from July 2019 to January 2020 (main sample, before the Corona pandemic spread exponentially in Germany) and from June 2020 to August 2020 (independent retest sample with a 2 weeks retest interval, timeframe with reduced Corona infections in Germany). Individuals took part in the online survey voluntarily, gave informed consent, and received course credit for participation. Besides being an adult, there were no prerequisites for study participation. The study was approved by the Ethics review board of the University of Potsdam (no. 2/2019, no. 51/ 2019 , no. 22/2020). The complete data set was used for validation of another OCD screening tool as well [17].

\section{Instruments}

Zohar-Fineberg obsessive compulsive screen (ZF-OCS; (7, 13))

We used the translation of the 5-item ZF-OCS published by the German Association for Psychiatry, Psychotherapy and Psychosomatics [7]. The screening is deemed positive if a person answers at least one of the five ZFOCS items positively. In the retest sample, we decided to add an item on individual impairment. As proposed by two previous publications $[10,14]$, we decided to add the item "Do these problems trouble you?" (in German "Beeinträchtigen Sie diese Probleme?"). In this case, the screening is deemed positive if a person answers at least one of the five ZF-OCS items positively, and if he or she perceives being impaired [7]. Therefore, sum scores ranged between 0 and 5 resp. 6 .

\section{Dimensional obsessive-compulsive scale (DOCS; $(2,18)$ )}

The DOCS covers the four main symptom dimensions of OCD: contamination, responsibility for harm, symmetry and thoughts that are perceived as unacceptable. Regarding each dimension, participants also indicate severity (i.e., time expenditure, avoidance behavior, distress, functional interference and difficulties in refraining). The items are rated on a 5-point Likert scale 
from zero (no symptoms) to four (extreme symptoms). Factor analysis revealed a stable four-factor structure complemented by a general OCD factor [2]. Evidence of stability, construct validity and diagnostic accuracy was provided as well $[2,19]$. The German translation displayed excellent reliability $(\alpha=.91)$, satisfactory to good construct validity and satisfactory diagnostic accuracy [18]. In the current study, the DOCS showed excellent internal consistency $(\alpha=.91)$.

\section{Obsessive-compulsive inventory-revised (OCI-R; $(20,21))$}

The OCI- $R$ is an 18 -item questionnaire on common OCD symptoms. Its items are rated on a 5-point Likert scale from zero (not at all) to four (very much). The OCI-R comprises six subscales on washing, checking, ordering, obsessing, neutralizing and hoarding. Research supported its six-factorial structure in a German clinical sample [21]. Foa and colleagues [20] reported excellent test-retest reliability. Strong support was found for its divergent [21] and convergent validity [22]. In the present study, it showed good internal consistency $(\alpha=.88)$.

\section{Patient health Questionnaire-2 (PHQ-2; (23))}

The PHQ-2 is a brief screening tool for symptoms of depression. Both items are rated on a 4-point Likert scale ranging from zero (not at all) to three (nearly every day). Evidence of its construct validity was provided by an association with risk factors of depression in the general population [23]. In the present study, the 2-item PHQ-2 yielded acceptable internal consistency $(\alpha=.78)$.

\section{Generalized anxiety disorder Scale-7 (GAD-7; $(24,25))$}

The GAD-7 screens for anxiety symptoms. Its items are rated on a 4-point scale ranging from zero (not at all) to three (nearly every day). There is evidence of a onedimensional factor structure, for its construct validity $[25,26]$ and for diagnostic accuracy [24]. In the current study, the GAD-7 showed good internal consistency $(\alpha=.85)$.

\section{IIIness attitude scale-bodily preoccupation subscale (IAS-BP; (27))}

The IAS-BP is the 3-item subscale of the IAS on bodily preoccupations. The items are rated on a 5-point Likert scale ranging from zero (never) to four (mostly). Weck et al. [27] described the IAS-BP as a valid screening instrument for health anxiety, and provided evidence of its diagnostic accuracy, for convergent and for divergent validity [27, 28]. In the present study, the IAS-BP showed acceptable internal consistency $(\alpha=.78)$.

World Health Organization well-being index (WHO-5; (29)) The WHO-5 is a short measure on subjective wellbeing. It comprises five items rated on a 6-point scale from zero (at no time) to five (all of the time). Raw sum scores were converted to percentages by multiplying them by four. Brähler et al. [29] provided evidence of the instrument's reliability and construct validity. In the present study, the WHO-5 showed good internal consistency $(\alpha=.85)$.

\section{Data analysis}

Statistical analyses were performed using IBM SPSS Statistics 24, the level of significance was set at .05 for all analyses. If there were single missing values, they were replaced by individual means.

\section{Reliability}

As it is the more established statistic, we investigated Cronbach's $\alpha$ first. According to Bland and Altman [30], Cronbach's $\alpha$ should be interpreted as follows: .70-.80 acceptable, $>.80$ good, and $>.90$ excellent. Since Cronbach's $\alpha$ applies more to items that clearly measure the same construct, and the ZF-OCS refers to various OCD symptom dimensions, McDonald's $\omega$ constitutes an alternative statistic in our study [31]. For its calculation, we used the OMEGA macro and syntax (31). If McDonald's $\omega$ is $>.80$, it may be interpreted as good [32]. Furthermore, we calculated the corrected item-total correlations, whereby values $>.40$ may be interpreted as good [33].

Concerning retest reliability at 2 weeks, Pearson correlations and paired t-tests were calculated for the ZFOCS sum scores between both measurement times. Correlations were considered high if $r$ was $\geq .50$ [34].

\section{Convergent and divergent validity}

Pearson correlations were computed again, and considered high if they were $\geq .50$ [34]. To investigate whether correlations between the ZF-OCS and the convergent measures were significantly stronger than the correlations between the ZF-OCS and the divergent measures, $t$-tests for comparing dependent correlations were used [35].

\section{Results}

Participants in the main sample $(N=304)$ were on average 24.86 years old $(S D=6.93)$. They were mostly female (76.3\%, $n=232)$ students $(85.5 \%, n=260)$. Being asked for prior diagnoses given by a physician or psychologist, seven participants $(2.3 \%)$ self-reported having been diagnosed with OCD, and $n=52(17.1 \%)$ stated having been diagnosed with depression before. Concerning the ZFOCS, $28.9 \%(n=88)$ of the participants did not answer positively to any item, $28.9 \%(n=88)$ affirmed one item, $18.1 \%(n=55)$ affirmed two items, $15.1 \%(n=46)$ three items, $6.9 \%(n=21)$ four, and $2 \%(n=6)$ affirmed all five items (for means and standard deviations of all measures, see Table 1). 
Table 1 Means and Standard Deviations in the Main Sample

\begin{tabular}{llll}
\hline & $\boldsymbol{M}$ & SD & Range \\
\hline ZF-OCS & 1.48 & 1.34 & $0-5$ \\
OCI-R & 14.06 & 9.84 & $0-45$ \\
DOCS & 10.91 & 9.29 & $0-42$ \\
PHQ-2 & 1.74 & 1.40 & $0-6$ \\
GAD-7 & 5.79 & 4.12 & $0-20$ \\
IAS-BP & 3.54 & 2.38 & $0-12$ \\
WHO-5 & 49.77 & 18.76 & $8-96$ \\
\hline
\end{tabular}

The independent retest sample comprised $N=51$ adults with an average age of $M=25.67$ years $(S D=$ 5.29). $78.4 \%$ were female $(n=40)$, and most of them were students $(78.4 \%, n=40)$. There were no significant differences between the main and the retest samples regarding age and gender $(p>.05)$.

Whereas between 21.7 and $38.8 \%$ of the participants responded positively to at least one of the five OC items (main sample, see Table 2), 15.7\% $(n=8$, retest 1$)$ resp. $13.7 \%(n=7$, retest 2$)$ confirmed the impairment item.

\section{Reliability}

Cronbach's $\alpha$ lay between .53 (main sample), and .72 (retest sample $t_{2}, 6$ items, see Table 3 ), and only the latter value may be interpreted as acceptable [35]. McDonald's $\omega$ lay between .55 (main sample) and .69 (retest sample $t_{2}, 6$ items). The corrected item-total correlations were good (i.e., above .40) for item 2 on checking only (see Table 2).

Regarding retest reliability at 2 weeks, the ZF-OCS sum scores (5 items) did not change significantly $\left(M_{\mathrm{t} 1}=\right.$ 1.31, $S D_{\mathrm{t} 1}=1.32 ; M_{\mathrm{t} 2}=1.29, S D_{\mathrm{t} 2}=1.39 ; t(50) .227, p=$ .821). The Pearson correlation was $r_{\mathrm{t} 1, \mathrm{t} 2}=.898$, indicating a high correlation.

Similarly, the ZF-OCS sum scores including the item on impairment (6 items) did not change significantly during the retest interval $\left(M_{\mathrm{t} 1}=1.47, S D_{\mathrm{t} 1}=1.58 ; M_{\mathrm{t} 2}=\right.$ 1.43, $\left.S D_{\mathrm{t} 2}=1.63 ; t(50) .375, p=.709\right)$. The Pearson correlation was $r_{\mathrm{t} 1, \mathrm{t} 2}=.892$, indicating a high correlation as well.

\section{Convergent and divergent validity}

For the main sample, Pearson correlations between the German ZF-OCS and the convergent measures were high, i.e., $r=.64$ (DOCS), and $r=.61$ (OCI-R) respectively (for all correlations, see Table 4).

The Pearson correlations between the ZF-OCS and the divergent measures were lower, i.e., $r=.39$ (PHQ-2), $r=.29$ (IAS-BP), and $r=-.28$ (WHO-5). Nevertheless, the ZF-OCS and the GAD-7 were associated more strongly $(r=.52)$.
Most correlations with convergent measures were significantly stronger than the correlations with the divergent measures (Supplement 1) which did not apply to the GAD-7. In that case, the correlation between the GAD-7 and the DOCS $(r=.66)$ was similar, but significantly stronger than the correlation between the ZFOCS and the DOCS $(r=.64, p=.001)$.

\section{Discussion}

The Zohar-Fineberg Obsessive-Compulsive Screen (ZFOCS) is described as "one of the most useful sets of screening questions" ([10] , p. 7) for detecting obsessivecompulsive (OC) symptoms. Although it was adopted by clinical guidelines [7, 14], the American Psychiatric Association [36] recommends choosing from slightly different screening questions. At the same time, the APA [36] emphasizes the need for developing a psychometrically sound screening instrument for use in primary care, as one of the key goals in current OCD research. The present study is one of the few to investigate the psychometric properties of the ZF-OCS, aiming to provide general practitioners with empirical data on the usefulness of this brief scale.

First of all, we investigated the internal consistency of the instrument, which, regardless of which coefficient we used, was low. This result indicates that we were not able to measure one coherent construct. First, the ZFOCS covers the most prevalent OC symptom dimensions [2], ranging from obsessional thoughts, to washing/cleaning and checking compulsions through to symmetry. Since the OC symptom dimensions are rather heterogeneous [2], good or even excellent internal consistency of such a short scale may not be expected [33]. Patients may be affected, for example, by contamination obsessions and cleaning compulsions, but not by repugnant obsessional thoughts and neutralizing strategies [2]. Nevertheless, a screening tool should cover all dimensions roughly. Furthermore, low internal consistency may also result from poor item interrelatedness [37]. Thus, we examined the corrected item-total correlations of the ZF-OCS, whereby it was good for one item only. Moreover, the ZF-OCS is a brief instrument, and its small number of items may contribute to its low internal consistency [33]. Since screening instruments should enable rough estimations with minimal effort, their reliability is often lower than that of more detailed measures [33].

Despite low internal consistency, retest reliability of a questionnaire may be high if the underlying construct is temporally stable [33]. As our second survey suggested, retest reliability was indeed high, both with and without including the additional item on individual impairment. Nevertheless, since we cannot exclude a memory effect [33], future studies should cover larger retest periods. 
Table 2 Intercorrelations between the ZF-OCS items in the Main $(n=304)$ and Retest $(n=51)$ Samples

\begin{tabular}{|c|c|c|c|c|c|c|c|}
\hline Main Sample & Item 1 wash & Item 2 check & Item 3 thought & Item 4 time & Item 5 order & Item-total $^{a}$ & $\begin{array}{l}\text { Yes } \\
\%\end{array}$ \\
\hline Item 1 & 1 & $.270^{* *}$ & $.179^{* *}$ & .073 & $.146^{*}$ & .269 & 24.3 \\
\hline Item 2 & & 1 & $.243^{* *}$ & $.142^{*}$ & $.322^{* *}$ & .413 & 34.2 \\
\hline Item 3 & & & 1 & $.227^{* *}$ & .102 & .297 & 28.9 \\
\hline Item 4 & & & & 1 & $.137^{*}$ & .227 & 21.7 \\
\hline Item 5 & & & & & 1 & .286 & 38.8 \\
\hline $\begin{array}{l}\text { Retest } \\
\text { Time } 1\end{array}$ & Item 1 wash & Item 2 check & Item 3 thought & Item 4 time & Item 5 order & Item 6 impairment & \\
\hline Item 1 & 1 & .123 & .142 & .159 & $.312^{*}$ & .142 & \\
\hline Item 2 & & 1 & .173 & .056 & $.409^{* *}$ & $.289^{*}$ & \\
\hline Item 3 & & & 1 & $.429^{* *}$ & .095 & $.852^{* *}$ & \\
\hline Item 4 & & & & 1 & .165 & $.560^{* *}$ & \\
\hline Item 5 & & & & & 1 & .206 & \\
\hline Item 6 & & & & & & 1 & \\
\hline $\begin{array}{l}\text { Retest } \\
\text { Time } 2\end{array}$ & Item 1 wash & Item 2 check & Item 3 thought & Item 4 time & Item 5 order & Item 6 impairment & \\
\hline Item 1 & 1 & $.289^{*}$ & .104 & .240 & $.336^{*}$ & .206 & \\
\hline Item 2 & & 1 & $.432^{* *}$ & .215 & $.334^{* *}$ & $.368^{* *}$ & \\
\hline Item 3 & & & 1 & $.485^{* *}$ & .065 & $.739^{* *}$ & \\
\hline Item 4 & & & & 1 & .242 & $.551^{* *}$ & \\
\hline Item 5 & & & & & 1 & .245 & \\
\hline Item 6 & & & & & & 1 & \\
\hline
\end{tabular}

Notes. ${ }^{*} p<.05, * * 0.01$ (two-tailed)

${ }^{a}$ corrected item-total correlations

The high correlations of the ZF-OCS with established OCD measures (i.e., $[18,21])$ suggests its convergent validity. As expected, the ZF-OCS screening also showed evidence of discriminant validity, since it was correlated significantly less with short measures on depression [23], health anxiety [27] and health-related well-being [29]. However, it turned out to be difficult to disentangle OC symptoms from general anxiety as measured by the GAD-7 - a problem that appeared in previous studies on OCD instruments as well [2, 17, 18, 38]. The reason for this may be twofold. First, OCD is conceptually related to the anxiety disorders [39]. Second, the GAD-7 asks rather broad questions on anxiety and general distress (such as "Feeling afraid as if something awful might

Table 3 Reliability as Measured by Cronbach's a and McDonald's $\omega$

\begin{tabular}{lll}
\hline & $\boldsymbol{a}$ & $\boldsymbol{\omega}$ \\
\hline Main sample & .53 & .55 \\
Retest sample $t_{1}, 5$ items & .57 & .58 \\
Retest sample $t_{1}, 6$ items & .68 & .66 \\
Retest sample $t_{2}, 5$ items & .65 & .64 \\
Retest sample $t_{2}, 6$ items & .72 & .69 \\
\hline
\end{tabular}

happen") which may be easily confused with OC symptoms.

We further examined the effect of an additional item on impairment. We included the item in our retest survey, whereby the additional item increased internal consistency as described above. Although between 22 and $39 \%$ of the participants approved at least one of the first five ZF-OCS items, this was true for maximally $16 \%$ regarding perceived impairment. This supports the suggestion to assess the screening as positive only if a participant approved at least one of the five ZF-OCS items and perceives impairment [7] - a suggestion that also reflects the current diagnostic criteria [1].

Table 4 Bivariate Pearson Correlations between all Measures in the Main Sample

\begin{tabular}{lcccccc}
\hline & OCI-R & DOCS & PHQ-2 & GAD-7 & IAS-BP & WHO-5 \\
\hline ZF-OCS & $.61^{* *}$ & $.64^{* *}$ & $.39^{* *}$ & $.52^{* *}$ & $.29^{* *}$ & $-.28^{* *}$ \\
OCI-R & & $.75^{* *}$ & $.39^{* *}$ & $.57^{* *}$ & $.42^{* *}$ & $-.30^{* *}$ \\
DOCS & & & $.44^{* *}$ & $.66^{* *}$ & $.39^{* *}$ & $-.34^{* *}$ \\
PHQ-2 & & & & $.65^{* *}$ & .11 & $-.66^{* *}$ \\
GAD-7 & & & & $.32^{* *}$ & $-.58^{* *}$ \\
IAS-BP & & & & & $-.13^{*}$ \\
\hline Notes. ${ }^{*} p<.05,{ }^{* *} p<.01$ (two-tailed) & & &
\end{tabular}


The Institute for Quality and Efficiency in Health Care [40] defines "screening" as the examination of symptomfree (mostly healthy) persons for early recognition of diseases. Although Fineberg and colleagues [16] report excellent sensitivity and good specificity of the ZF-OCS in a dermatological sample, the German guideline authors indicate lower specificity due to their clinical experience with the scale [7]. Nevertheless, underrecognition is associated with undertreatment of OCD [12]. Therefore, false-positives may be acceptable here, as screening aims at initiating more detailed diagnostic procedures, referral to specialized treatment, thus counteracting possible chronification of the disease $[7,33]$. Nonetheless, future studies with patient groups should investigate the sensitivity and specificity of the ZF-OCS, using an established diagnostic interview on mental disorders. Furthermore, further research could investigate the ZF-OCS as a selfreport vs. a clinician-administered instrument, thus providing information on the most useful way to implement the scale.

\section{Conclusions}

The present study investigated the psychometric properties of the ZF-OCS as a screening tool for OC symptoms. Although internal consistency was improvable, retest reliability, and convergent and divergent validity were very good. Given that the ZF-OCS includes five items, and one item on individual impairment only, we regard it as a feasible measure for use in basic health care settings. Our results suggest that including the impairment item is useful. However, we suggest further investigation of the instrument's diagnostic accuracy in different samples (e.g., in specialized mental health care).

\begin{abstract}
Abbreviations
APA: American Psychiatric Association; DGPPN: German Association for Psychiatry, Psychotherapy and Psychosomatics; DOCS: Dimensional Obsessive-Compulsive Scale; GAD-7: Generalized Anxiety Disorder Scale-7; IAS-BP: IIIness Attitude Scale-Bodily Preoccupation Subscale; OCD: Obsessivecompulsive disorders; OCI-R: Obsessive-Compulsive Inventory-Revised; PHQ2: Patient Health Questionnaire-2; WHO-5: World Health Organization WellBeing Index; ZF-OCS: Zohar-Fineberg Obsessive-Compulsive Screen
\end{abstract}

\section{Supplementary Information}

The online version contains supplementary material available at https://doi. org/10.1186/s12888-021-03458-x.

Additional file 1: Supplement 1. T-tests for Comparing Dependent Correlation Coefficients.

\section{Acknowledgements}

We would like to thank Dr. Brian Bloch for editing the English.

\section{Authors' contributions}

Conceptualization: FK; Methodology and Analysis: FK, TP; Investigation: TP; Resources: FW; Writing - Original Draft: FK; Writing - Review \& Editing: FK, TP, FW; Supervision and Project Administration: FW. The author(s) read and approved the final manuscript.

\section{Funding}

This research did not receive any specific grant from funding agencies in the public, commercial, or not-for-profit sectors. We acknowledge the support of the Deutsche Forschungsgemeinschaft and Open Access Publishing Fund of University of Potsdam. Open Access funding enabled and organized by Projekt DEAL.

\section{Availability of data and materials}

The datasets used and/or analyzed during the current study are available from the corresponding author on reasonable request.

\section{Declarations}

Ethics approval and consent to participate

The study was approved by the Ethics review board of the University of Potsdam (no. 2/2019, no. 51/2019, no. 22/2020), and participants gave informed consent. All methods were carried out in accordance with relevant guidelines and regulations.

\section{Consent for publication}

Not applicable.

\section{Competing interests}

The authors declare that they have no competing interests.

Received: 22 February 2021 Accepted: 31 August 2021

Published online: 11 September 2021

\section{References}

1. American Psychiatric Association [APA]. Diagnostic and statistical manual of mental disorders. 5th ed. Washington: American Psychiatric Publishing; 2013.

2. Abramowitz JS, Deacon BJ, Olatunji BO, Wheaton MG, Berman NC, Losardo $D$, et al. Assessment of obsessive-compulsive symptom dimensions: development and evaluation of the dimensional obsessive-compulsive scale. Psychol Assess. 2010;22(1):180-98. https://doi.org/10.1037/a0018260.

3. Kessler RC, Petukhova M, Sampson NA, Zaslavsky AM, Wittchen HU. Twelvemonth and lifetime prevalence and lifetime morbid risk of anxiety and mood disorders in the United States. Int J Methods Psychiatr Res. 2012; 21(3):169-84. https://doi.org/10.1002/mpr.1359.

4. Jacobi F, Höfler M, Siegert J, Mack S, Gerschler A, Scholl L, et al. Twelvemonth prevalence, comorbidity and correlates of mental disorders in Germany: the mental health module of the German health interview and examination survey for adults (DEGS1-MH). Int J Methods Psychiatr Res. 2014;23(3):304-19. https://doi.org/10.1002/mpr.1439.

5. Koran LM. OCD and quality of life. In: Fineberg NA, Marazziti D, Stein D, editors. Obsessive-compulsive disorder: a practical guide. London: Martin Dunitz; 2001. p. 51-60.

6. Huppert JD, Simpson HB, Nissenson KJ, Liebowitz MR, Foa EB. Quality of life and functional impairment in obsessive-compulsive disorder: a comparison of patients with and without comorbidity, patients in remission, and healthy controls. Depress Anxiety. 2009;26(1):39-45. https://doi.org/10.1002/da.20506.

7. Deutsche Gesellschaft für Psychiatrie und Psychotherapie, Psychosomatik und Nervenheilkunde [German Association for Psychiatry, Psychotherapy and Psychosomatics; DGPPN]. S3-Leitlinie Zwangsstörungen [S3-Guideline for OCD]. 2014. https://www.awmf.org/uploads/tx_szleitlinien/ 038_0171_S3 Zwangsst\%C3\%B6rungen_2013-abgelaufen.pdf. Accessed 15 Apr 2020.

8. Wahl K, Kordon A, Kuelz KA, Voderholzer U, Hohagen F, Zurowski B. Obsessive-compulsive disorder (OCD) is still an unrecognised disorder: a study on the recognition of OCD in psychiatric outpatients. Eur Psychiatry. 2010;25(7):374-7. https://doi.org/10.1016/j.eurpsy.2009.12.003.

9. Olariu E, Forero CG, Castro-Rodriguez Jl, Rodrigo-Calvo MT, Álvarez P, Martín-López LM, et al. Detection of anxiety disorders in primary care: a meta-analysis of assisted and unassisted diagnoses. Depress Anxiety. 2015; 32(7):471-84. https://doi.org/10.1002/da.22360.

10. Zohar J. Obsessive compulsive disorder: current science and clinical practice. Hoboken, New Jersey: Wiley; 2012. https://doi.org/10.1002/9781119941125.

11. Szentagotai-Tatar A, Nechita DM, Miu AC. Shame in anxiety and obsessivecompulsive disorders. Current Psychiatry Reports. 2020;22(4):16. https://doi. org/10.1007/s11920-020-1142-9. 
12. Veldhuis J, Dieleman JP, Wohlfahrt T, Storosum JG, van den Brink W, Sturkenboom CJ, et al. Incidence and prevalence of "diagnosed OCD" in a primary care, treatment seeking, population. Int J Psychiatry Clin Pract. 2012; 16(2):85-92. https://doi.org/10.3109/13651501.2011.617454.

13. Fineberg NA, Roberts A. Obsessive compulsive disorder: a twenty-first century perspective. In: Fineberg NA, Marazziti D, Stein D, editors. Obsessive-compulsive disorder: a practical guide. London: Martin Dunitz; 2001. p. 1-13. https://doi.org/10.3109/9780203212899.

14. National Institute for Health and Care Excellence [NICE]. Obsessivecompulsive disorder (NICE Guideline 31). 2016. https://www.nice.org.uk/ guidance/cg31/evidence. Accessed 15 Apr 2020.

15. Sheehan DV, Lecrubier $Y$, Sheehan $\mathrm{KH}$, Amorim P, Janavs J, Weiler E, et al. The Mini-International Neuropsychiatric Interview (MINI): the development and validation of a structured diagnostic psychiatric interview for DSM-IV and ICD-10. J Clin Psychiatry. 1998;59:34.

16. Fineberg NA, O'Doherty C, Rajagopal S, Reddy K, Banks A, Gale TM. How common is obsessive-compulsive disorder in a dermatology outpatient clinic? J Clin Psychiatry. 2003;64(2):152-5. https://doi.org/10.4 088/jcp.v64n0207

17. Kühne F, Paunov T, Abramowitz JS, Fink-Lamotte J, Hansen B, Kvale G, et al. Screening for obsessive-compulsive symptoms: validation of the dimensional obsessive-compulsive scale - English and German short forms. J Obsess Compuls Relat Disord. 2021;29:100625. https://doi.org/10.1016/j. jocrd.2021.100625

18. Fink-Lamotte J, Jahn I, Stierle C, Kühne F, Lincoln T, Stengler K, et al. Die Validierung der dimensional obsessive-compulsive scale (DOCS) an einer deutschsprachigen Stichprobe [validation of the dimensional obsessivecompulsive scale (DOCS) in a German sample]. Verhaltenstherapie. 2020; 31(2):119-31. https://doi.org/10.1159/000510093.

19. Thibodeau MA, Leonard RC, Abramowitz JS, Riemann BC. Secondary psychometric examination of the dimensional obsessive-compulsive scale: classical testing, item response theory, and differential item functioning. Assessment. 2015;22(6):681-9. https://doi.org/10.1177/1073191114559123.

20. Foa EB, Huppert JD, Leiberg $S$, Langner R, Kichic R, Hajcak $G$, et al. The obsessive-compulsive inventory: development and validation of a short version. Psychol Assess. 2002;14(4):485-96. https://doi.org/10.1037/1040-3 590.14.4.485.

21. Gönner S, Leonhart R, Ecker W. The obsessive-compulsive inventory-revised (OCl-R): validation of the German version in a sample of patients with OCD, anxiety disorders, and depressive disorders. J Anxiety Disord. 2008;22(4):734-49. https:/doi.org/10.1016/j.janxdis.2007.07.007.

22. Gönner S, Leonhart R, Ecker W. Das Zwangsinventar OCI-R - die deutsche version des obsessive-compulsive inventory-revised - ein kurzes Selbstbeurteilungsinstrument zur mehrdimensionalen Messung von Zwangssymptomen [the German version of the obsessive-compulsive inventory-revised: a brief self-report measure for the multidimensional assessment of obsessive-compulsive symptoms]. Psychotherapie, Psychosomatik, Medizinische Psychologie. 2007. https://doi.org/10.1055/s $-2007-970894$

23. Löwe B, Wahl I, Rose M, Spitzer C, Glaesmer H, Wingenfeld K, et al. A 4-item measure of depression and anxiety: validation and standardization of the patient health Questionnaire-4 (PHQ-4) in the general population. J Affect Disord. 2010;122(1-2):86-95. https://doi.org/10.1016/j.jad.2009.06.019.

24. Spitzer RL, Kroenke K, Williams JB, Löwe B. A brief measure for assessing generalized anxiety disorder: the GAD-7. Arch Intern Med. 2006;166(10): 1092-7. https://doi.org/10.1001/archinte.166.10.1092.

25. Löwe B, Decker O, Müller S, Brähler E, Schellberg D, Herzog W, et al. Validation and standardization of the generalized anxiety disorder screener (GAD-7) in the general population. Med Care. 2008;46(3):266-74. https://doi. org/10.1097/MLR.0b013e318160d093.

26. Hinz A, Klein AM, Brähler E, Glaesmer H, Luck T, Riedel-Heller SG, et al. Psychometric evaluation of the generalized anxiety disorder screener GAD7, based on a large German general population sample. J Affect Disord. 2017;210:338-44. https://doi.org/10.1016/j.jad.2016.12.012.

27. Weck F, Bleichhardt G, Hiller W. Screening for hypochondriasis with the illness attitude scales. J Pers Assess. 2010;92(3):260-8. https://doi.org/10.1 080/00223891003670216.

28. Höfling V, Weck F. Assessing bodily preoccupations is sufficient: clinically effective screening for hypochondriasis. J Psychosom Res. 2013;75(6):526-31. https://doi.org/10.1016/j.jpsychores.2013.10.011.
29. Brähler E, Mühlan H, Albani C, Schmidt S. Teststatistische Prüfung und Normierung der deutschen Versionen des EUROHIS-QOL Lebensqualitätindex und des WHO-5 Wohlbefindens-index [testing and standardization of the German version of the EUROHIS-QOL and WHO-5 quality of life indices]. Diagnostica. 2007;53(2):83-96. https://doi.org/10.1026/0012-1924.53.2.83.

30. Bland JM, Altman DG. Cronbach's alpha. BMJ (Clinical Research Ed.). 1997; https://doi.org/10.1136/bmj.314.7080.572.

31. Hayes AF, Coutts JJ. Use omega rather than Cronbach's alpha for estimating reliability. But .... Commun Methods Measures. 2020;14:1-24.

32. Feißt M, Hennigs A, Heil J, Moosbrugger H, Kelava A, Stolpner I, et al. Refining scores based on patient reported outcomes-statistical and medical perspectives. BMC Med Res Methodol. 2019;19(1):167. https://doi.org/10.11 86/s12874-019-0806-9.

33. Moosbrugger $\mathrm{H}$, Kelava A. Testtheorie und Fragebogenkonstruktion [test theory and questionnaire construction]. 2nd ed. Berlin: Springer; 2012. https://doi.org/10.1007/978-3-642-20072-4.

34. Bortz J, Döring N. Forschungsmethoden und evaluation für human- und Sozialwissenschaftler [research methods and evaluation for human and social scientists]. Berlin: Springer; 2006. https://doi.org/10.1007/978-3-54 0-33306-7.

35. Weaver B, Wuensch KL. SPSS and SAS programs for comparing Pearson correlations and OLS regression coefficients. Behav Res Methods. 2013;45(3): 880-95. https://doi.org/10.3758/s13428-012-0289-7.

36. American Psychiatric Association [APA]. Practice guideline for the treatment of patients with obsessive-compulsive disorder. 2007. http//www.psych.org/ psych_pract/treatg/pg/prac_guide. cfm. Accessed 29 Oct 2020.

37. Tavakol M, Dennick R. Making sense of Cronbach's alpha. Int J Med Educ. 2011;2:53-5. https://doi.org/10.5116/ijme.4dfb.8dfd.

38. Eilertsen T, Hansen B, Kvale G, Abramowitz JS, Holm SE, Solem S. The dimensional obsessive-compulsive scale: development and validation of a short form (DOCS-SF). Front Psychol. 2017;5(8):1503. https://doi.org/10.3389/ fpsyg.2017.01503.

39. Abramowitz JS. Presidential address: are the obsessive-compulsive related disorders related to obsessive-compulsive disorder? A critical look at DSM5's new category. Behav Ther. 2018;49(1):1-11. https://doi.org/10.1016/j. beth.2017.06.002.

40. Institut für Qualität und Wirtschaftlichkeit im Gesundheitswesen [Institute for Quality and Efficiency in Health Care; IQWIG]. Glossar zu den allgemeinen Methoden [Glossary of general methods]. 2008. https://www. iqwig.de/download/Glossar_Version_1_0_zu_den_Allgemeinen_ Methoden_Version_3_0.pdf. Accessed 26 Oct 2020.

\section{Publisher's Note}

Springer Nature remains neutral with regard to jurisdictional claims in published maps and institutional affiliations.

Ready to submit your research? Choose BMC and benefit from

- fast, convenient online submission

- thorough peer review by experienced researchers in your field

- rapid publication on acceptance

- support for research data, including large and complex data types

- gold Open Access which fosters wider collaboration and increased citations

- maximum visibility for your research: over $100 \mathrm{M}$ website views per year

At $\mathrm{BMC}$, research is always in progress.

Learn more biomedcentral.com/submissions 